\title{
A REMARK ON THE CONVERGENCE OF THE TIKHONOV REGULARIZATION WITHOUT MONOTONICITY
}

\author{
ABDELlaTIF MOUDAFI
}

\begin{abstract}
Relying on an idea of T. Pennanen, we use a localized version of maximal hypomonotonicity, and prove that it is enough to guarantee the local convergence of the Tikhonov regularization. In the process, we revisit this method which together with the help of the analysis developed by T. Pennanen allows us to obtain the desired result.
\end{abstract}

Mathematics subject classification (2000): 49J53, 65K10, 49M37, 90C25.

Key words and phrases: monotone and hypomonotone operators, Tikhonov regularization.

\section{REFERENCES}

[1] H. ATtouch, D. AzÉ, Approximation and regularization of arbitrary functions in Hilbert spaces by the Lasry-Lions method, Ann. Inst. Henri Poincaré, Anal. Non Linéaire 10, No. 3, (1993), pp. 289-312.

[2] A. N. IUSEM, T. PenNANen, B. F. Svaiter, Inexact variants of the proximal point algorithm without monotonicity., SIAM Journal on Optimization 13 4, (2003), 1080-1097

[3] T. Pennanen, Local convergence of the proximal point algorithm and multiplier methods without monotonicity, Mathematics of Operations Research, No. 27 (2002), pp. 170-191.

[4] R. T. Rockafellar, R. J.-B WeTs, Variational analysis. Springer, Belin (1998). 